\title{
Food Physical Chemistry and Biophysical Chemistry
}

\author{
I. C. Baianu \\ FSHN and NPRE Departments, University of Illinois at Urbana-Champaign, \\ AFC-NMR \& FT-NIR Microspectroscopy Facility, Urbana IL 61801, USA \\ Email: ibaianu@illinois.edu
}

\begin{abstract}
Food physical chemistry is considered to be a branch of Food chemistry [1] [2] concerned with the study of both physical and chemical interactions in foods in terms of physical and chemical principles applied to food systems, as well as the applications of physical/chemical techniques and instrumentation for the study of foods.[3] [4] [5] [6] This field encompasses the "physiochemical principles of the reactions and conversions that occur during the manufacture, handling, and storage of foods"[7]. Two rapidly growing, related areas are Food Biotechnology and Food Biophysical Chemistry.
\end{abstract}

Keywords: Food chemistry, Physical Chemistry, Biophysical Chemistry applicationsto food systems; physical and chemical principles applied to food systems, physical/chemical techniques and instrumentation utilized for the study of foods; physical and chemical aspects of food processing; reactions in foods; chemical analysis and related physical methods of analysis.

PACS: 82.70.-yxx; 82.80.-dxx ;44-XX; 47-XX; 47.50.+d; 47.55.-txx.; 47.70.-nxx;82.20.-wxx;82.56.-bxx; 82.60.-sxx.

\section{INTRODUCTION}

Food physical chemistry concepts are often drawn from rheology, theories of transport phenomena, physical and chemical thermodynamics, chemical bonds and interaction forces, quantum mechanics and reaction kinetics, biopolymer science, colloidal interactions, nucleation, glass transitions and freezing,[8] [9] disordered/ noncrystalline solids. Techniques utilized range widely from dynamic rheometry, optical microscopy, electron microscopy, AFM, light scattering, X-ray diffraction/neutron diffraction,[10] to MRI, spectroscopy (NMR,[11] FTNIR/IR, NIRS, Raman, ESR and EPR,[12] [13] CD/VCD,[14] Fluorescence, FCS [15] [16] [17] [18] [19] HPLC, GC-MS,[20] [21] and other related analytical techniques. Understanding food processes and the properties of foods requires knowledge of physical chemistry and how it applies to specific foods and food processes. Food physical chemistry is essential for improving the quality of foods, their stability and food product development. Because food science is a multi-disciplinary field, food physical chemistry is being developed through interactions with other areas of food chemistry and food science, such as: food analytical chemistry, food process engineering/food processing, food and bioprocess technology, food extrusion, food quality control, food packaging, food biotechnology and food microbiology.

\section{TOPICS IN FOOD PHYSICAL CHEMISTRY}

\subsection{Topic Groups and Categories}

The following are examples of topics in Food Physical Chemistry that are of interest to both Food Industry and Food Science:

- Water in foods

- Local structure in liquid water

- Micro-crystallization in icecream emulsions

- Dispersion and surface-adsorption processes in foods; Food Dispersions

- Water and protein activities

- Food hydration and shelf-life

- Hydrophobic interactions in foods

- Hydrogen bonding and ionic interactions in foods

- Disulfide bond breaking and formation in foods

- Food dispersions

- Structure-functionality in foods 
- Food micro- and nano- structure

- Food gels and gelling mechanisms

- Cross-linking in foods

- Starch gelatinization and retrogradation

- Physico-chemical modification of carbohydrates

- Physico-chemical interactions in food formulations

- Freezing effects on foods and freeze concentration of liquids

- Glass transition in wheat gluten and wheat doughs

- Drying of foods and crops

- Rheology of wheat doughs, cheese and meat

- Rheology of extrusion processes

- Food enzyme kinetics

- Immobilized enzymes and cells

- Microencapsulation

- Carbohydrates structure and interactions with water and proteins

- Maillard browning reactions

- Lipids structures and interactions with water and food proteins

- Food proteins structure, hydration and functionality in foods

- Food protein denaturation

- Food enzymes and reaction mechanisms

- Vitamin interactions and preservation during food processing

- Interaction of salts and minerals with food proteins and water

- Color determinations and food grade coloring

- Flavors and sensorial perception of foods

- Properties of food additives

\begin{tabular}{|l|l|}
\hline \multicolumn{2}{|c|}{ Origin } \\
\hline Ice cream or gelato in Rome, Italy \\
\hline Alternative name(s) & Gelato, sorbet, frozen custard \\
\hline \multicolumn{2}{|c|}{ Dish details } \\
\hline Course served & Dessert \\
\hline Main ingredient(s) & Milk/Cream, water ice, sugar \\
\hline
\end{tabular}

Figure 1.1. Examples of solid and glassy (non-crustalline) food dispersions that also includes ice microcrystals. 


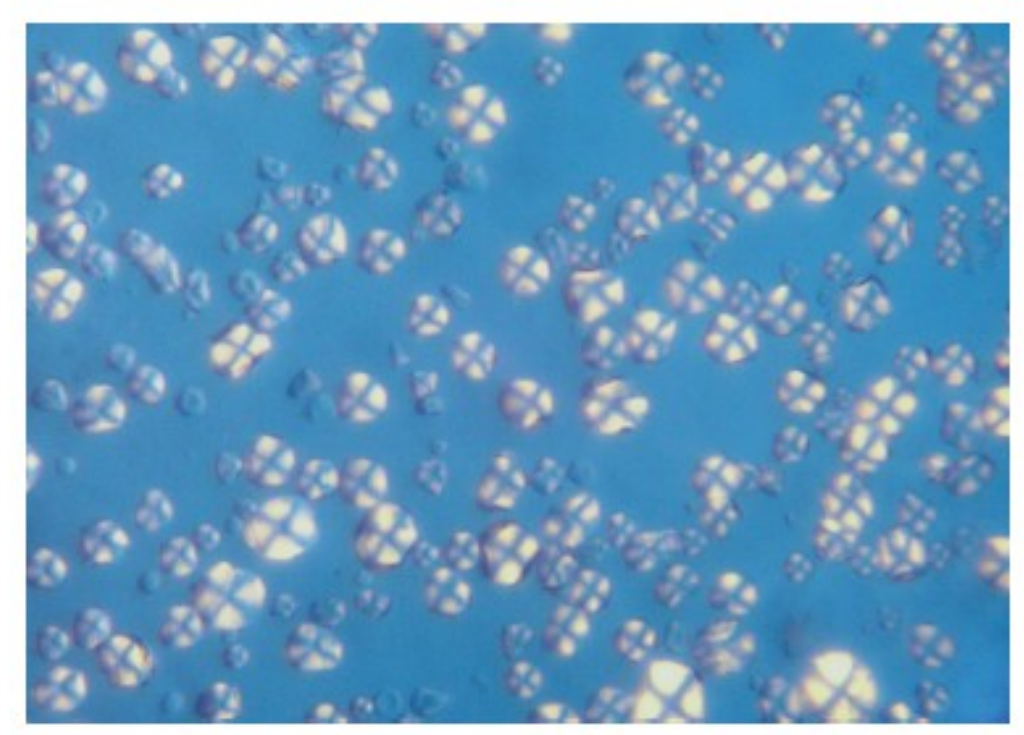

\section{Starch, 800x magnified, under polarized light}

Figure 1.2. An example of a non-crystalline and non-Newtonian, but textured food system; this is usually classified as a gel that has a complex, hydrated network structure.

\subsection{Paracrystal Theory and Convolution Algebra}

A detailed example of physical chemistry applications to heterogeneous systems such as solid foods and food dispersions is the paracrystal theory application to X-ray and neutron scattering data from such complex systems.

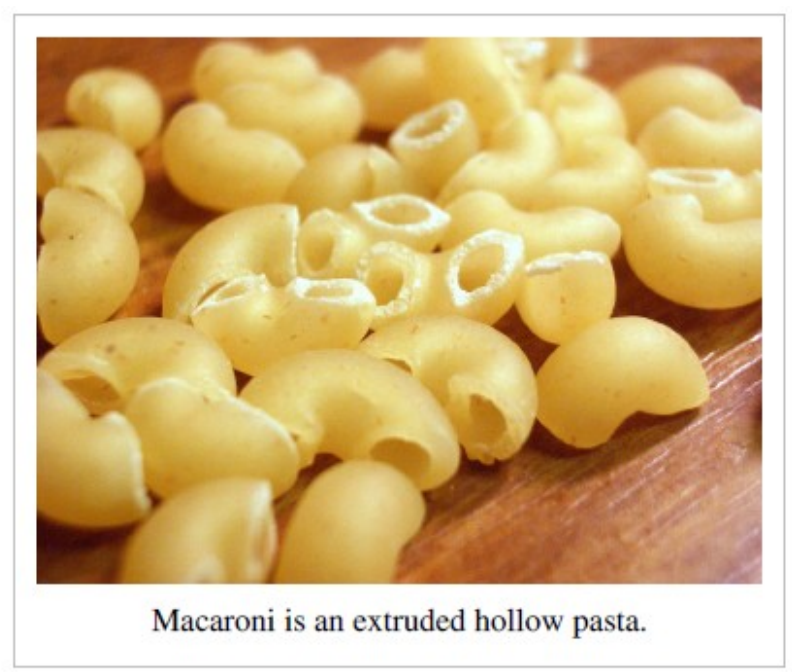

Figure 2.1. Example of an important and major, but common, food ingredient that includes paracrystals-in this case a mixture of crystalline and non-crystalline structures.

As reported in a recent publication [21], the general theory of scattering by partially ordered, atomic or molecular, structures in terms of paracrystals and lattice convolutions was formulated by Hosemann and Bagchi in [145] using basic techniques of Fourier analysis and convolution products. A natural generalization of such molecular, partial 
symmetries and their corresponding analytical versions involves convolution algebras - a functional/distribution $[247,248]$ based theory that we will discuss in the context of a more general and original concept of a convolution-algebroid of an extended symmetry groupoid of a paracrystal, of any molecular or nuclear system, or indeed, any quantum system, in general. Such applications also include quantum fields theories, and local quantum net configurations that are endowed with either partially disordered or 'completely' ordered structures, as well as in the graded, or super-algelbroid extension of these concepts for very massive structures such as stars and black holes treated by quantum gravity theories.

A statistical analysis linked to structural symmetry and scattering theory considerations shows that a real paracrystal can be defined by a three dimensional convolution polynomial with a semi-empirically derived composition law, *, [146]. As was shown in $[13,14]$ - supported with computed specific examples - several systems of convolution can be expressed analytically, thus allowing the numerical computation of $X$-ray, or neutron, scattering by partially disordered layer lattices via complex Fourier transforms of one-dimensional structural models using fast digital computers. The range of paracrystal theory applications is however much wider than the one-dimensional lattices with disorder, thus spanning very diverse non-crystalline systems, from metallic glasses and spin glasses to superfluids, high-temperature superconductors, and extremely hot anisotropic plasmas such as those encountered in controlled nuclear fusion (for example, JET) experiments. Other applications - as previously suggested in [12] - may also include novel designs of 'fuzzy' quantum machines and quantum computers with extended symmetries of quantum state spaces.

\subsubsection{Convolution product of groupoids and the convolution algebra of functions}

From a purely mathematical perspective, Alain Connes introduced the concept of a $C^{*}$-algebra of a (discrete) group (see, e.g., [91]). The underlying vector space is that of complex valued functions with finite support, and the multiplication of the algebra is the fundamental convolution product which it is convenient for our purposes to write slightly differently from the common formula as

and *-operation

$$
(f * g)(z)=\sum_{x y=z} f(x) g(y)
$$

$$
f(x)=\overline{f\left(x^{-1}\right)}
$$

The more usual expression of these formulas has a sum over the elements of a selected group. For topological groups, where the underlying vector space consists of continuous complex valued functions, this product requires the availability of some structure of measure and of measurable functions, with the sum replaced by an integral. Notice also that this algebra has an identity, the distribution function $\delta_{1}$, which has value one on the identity 1 of the group, and has zero value elsewhere. Given this convolution/distribution representation that combines crystalline ('perfect' or global-group, and/or group-like symmetries) with partial symmetries of paracrystals and glassy solids on the one hand, and also with non-commutative harmonic analysis [182] on the other hand, we propose that several extended quantum symmetries can be represented algebraically in terms of certain structured groupoids, their

$C^{*}$-convolution quantum algebroids, paragroup/quantized groups and/or other more general mathematical structures that will be introduced in this report. It is already known that such extensions to groupoid and algebroid/coalgebroid symmetries require also a generalization of non-commutative harmonic analysis which involves certain Haar measures, generalized Fourier - Stieltjes transforms and certain categorical duality relationships representing very general mathematical symmetries as well. A very promising approach to nonlinear (anharmonic) analysis of aperiodic quantum systems represented by rigged Hilbert space bundles may involve the computation of representation coefficients of Fourier - Stieltjes groupoid transforms.

Currently, however, there are several important aspects of quantum dynamics left out of the invariant, simplified picture provided by group symmetries and their corresponding representations of quantum operator algebras [126]. An alternative approach proposed in [140] employs differential forms to find such symmetries. Physicists deal often with such problems in terms of either spontaneous symmetry breaking or approximate symmetries that require underlying assumptions or ad-hoc dynamic restrictions that have a phenomenological basisl. A well-studied example of this kind is that of the dynamic Jahn -Teller effect and the corresponding 'theorem' (Chapter 21 on pp. 807 - 831, as well as p. 735 of [1]) which in its simplest form stipulates that a quantum state with electronic non-Kramers degeneracy may be unstable against small distortions of the surroundings, that would lower the symmetry of the crystal field and thus lift the degeneracy (i.e., cause an observable splitting of the corresponding energy levels). This effect occurs in certain paramagnetic ion systems via dynamic distortions of the crystal field symmetries around paramagnetic or high-spin centers by moving ligands that are diamagnetic. The established physical explanation is 
that the Jahn - Teller coupling replaces a purely electronic degeneracy by a vibronic degeneracy (of exactly the same symmetry!). The dynamic, or spontaneous breaking of crystal field symmetry (for example, distortions of the octahedral or cubic symmetry) results in certain systems in the appearance of doublets of symmetry $\gamma_{3}$ or singlets of symmetry $\gamma_{1}$ or $\gamma_{2}$. Such dynamic systems could be locally expressed in terms of symmetry representations of a Lie algebroid, or globally in terms of a special Lie (or Lie - Weinstein) symmetry groupoid representations that can also take into account the spin exchange interactions between the Jahn - Teller centers exhibiting such quantum dynamic effects. Unlike the simple symmetries expressed by group representations, the latter can accommodate a much wider range of possible or approximate symmetries that are indeed characteristic of real, molecular systems with varying crystal field symmetry, as for example around certain transition ions dynamically bound to ligands in liquids where motional narrowing becomes very important. This well known example illustrates the importance of the interplay between symmetry and dynamics in quantum processes. Therefore, the various interactions and interplay between the symmetries of quantum operator state space geometry and quantum dynamics at various levels leads to both algebraic and topological structures that are variable and complex, well beyond symmetry groups and well-studied group algebras (such as Lie algebras, see for example [126]).

This important example is drawn from the general theory of scattering by partially ordered, atomic or molecular, structures in terms of paracrystals and lattice convolutions that was formulated in [145] using basic techniques of Fourier analysis and convolution products. Further specific applications of the paracrystal theory to $X$-ray scattering, based on computer algorithms, programs and explicit numerical computations, were subsequently developed by the first author [13] for one-dimensional paracrystals, partially ordered membrane lattices [14] and other biological structures with partial structural disorder [16]. Such biological structures, 'quasi-crystals', and the paracrystals, in general, provide rather interesting physical examples of extended symmetries (cf. [144], [new IJRRAS]).

\section{RELATED FIELDS TO FOOD PHYSICAL CHEMISTRY:}

- Food chemistry

- Food physics and Rheology

- Food technology, Food engineering, Food safety and Food biotechnology

- Agricultural biotechnology

- Biophysical chemistry

- Physical chemistry

- Chemical physics

- Chemical engineering

- Spectroscopy-applied

- Intermolecular forces

- Nanotechnology and nanostructures

- Molecular dynamics

- Surface chemistry and Van der Waals forces

- Chemical reactions and Reaction chemistry

- Quantum chemistry

- Quantum genetics

- Bioorganic chemistry

- Polymer chemistry

- Biochemistry and Biological chemistry

- Molecular models of DNA and Molecular modelling of proteins and viruses

- Enzymology

- Protein-protein interactions

- Biomembranes

- Complex system biology

- Systems biology

- Integrative biology

- Mathematical biophysics

- Genomics, Proteomics, Interactomics, Structural bioinformatics and Cheminformatics

- Immobilized cells and enzymes

- Microencapsulation of food additives and vitamins, etc. 
- Plant biology and Crop sciences

- Animal sciences

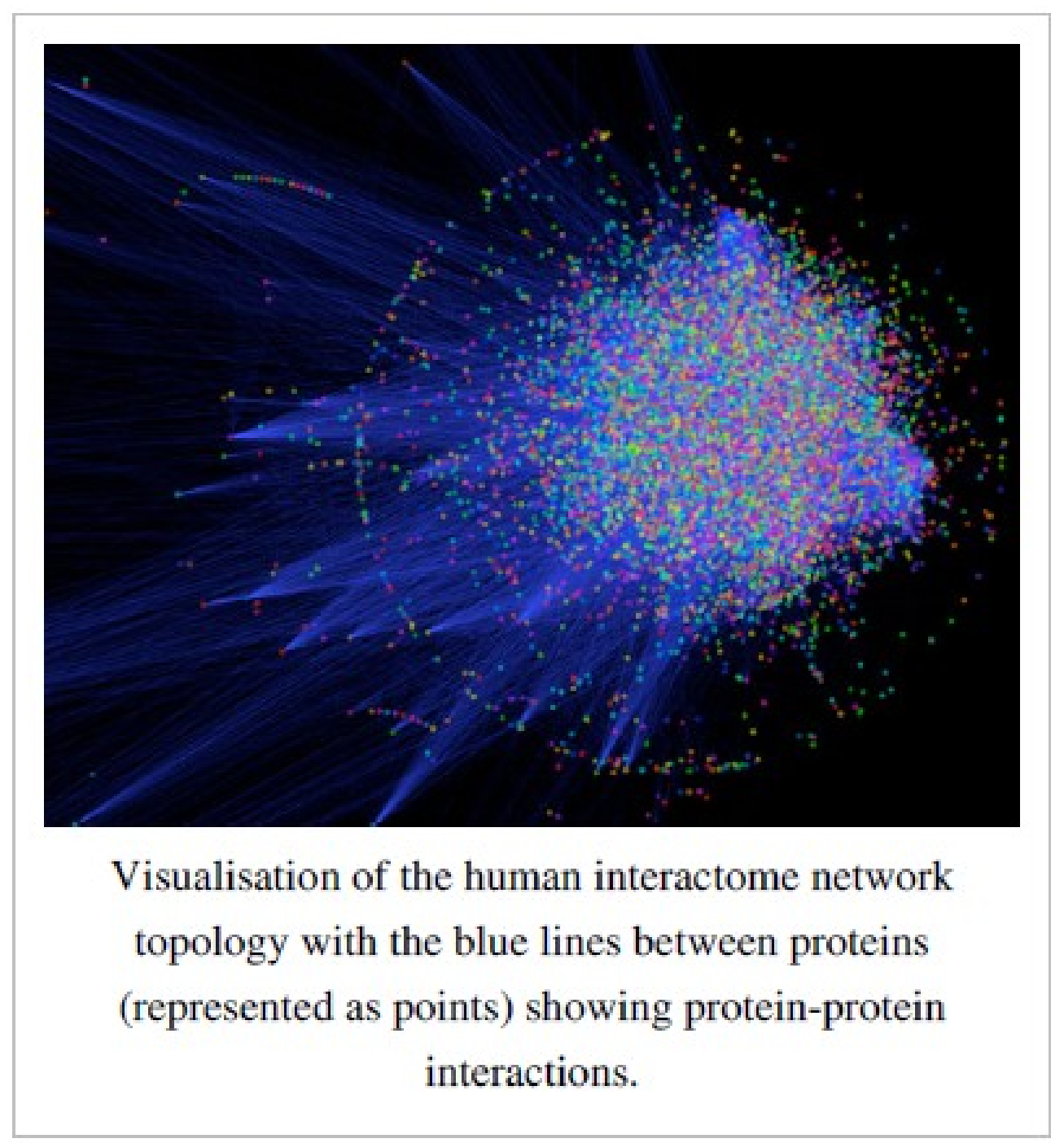

Figure 3.1. Visualisation of the human interactome network topology with the blue lines between proteins (represented as points) showing protein-protein interactions. 
4. TECHNIQUES GALLERY: High-Field NMR, CARS / Raman Spectroscopy, Fluorescence Confocal Microscopy and Hyperspectral Imaging
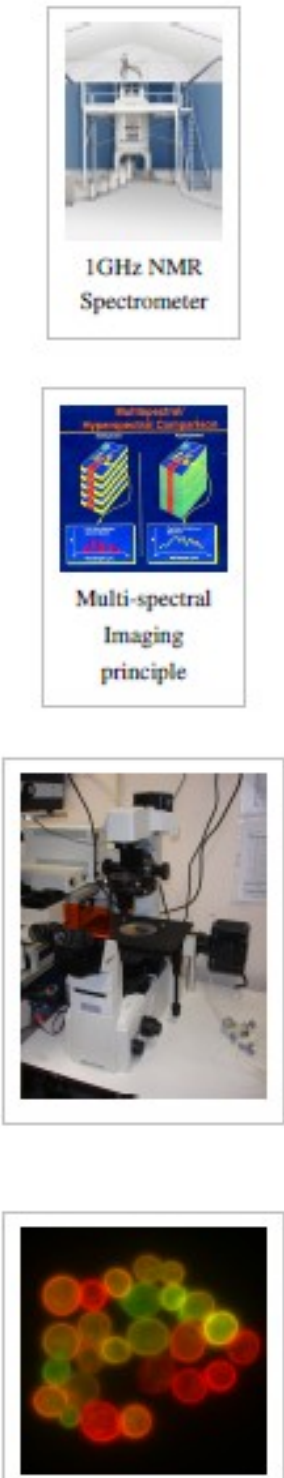

Yeast membrane protein imaging

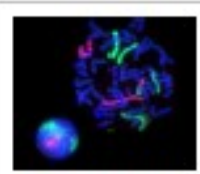

Red blood cell pathology
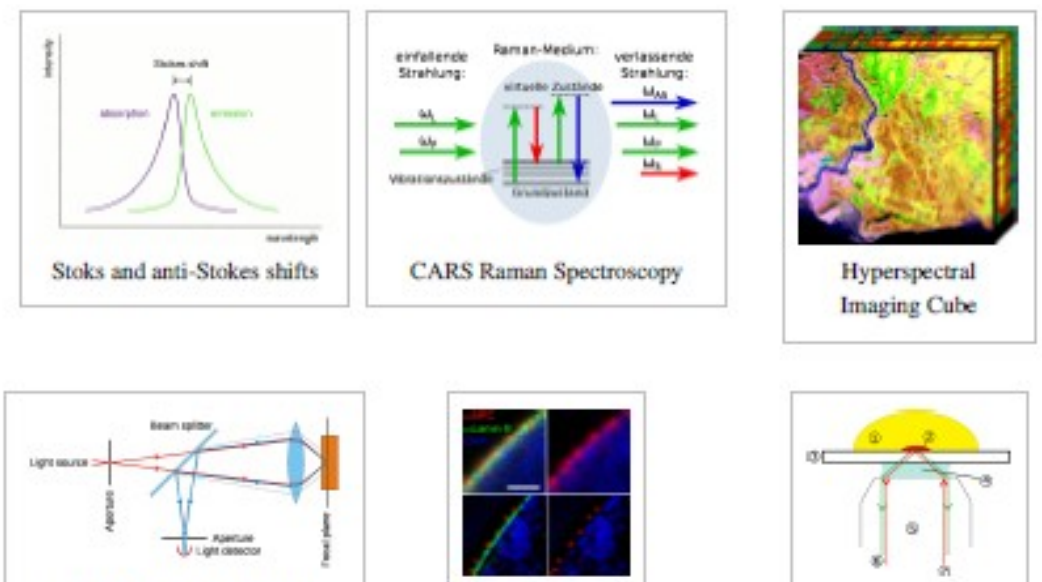

Confocal Imaging Principle
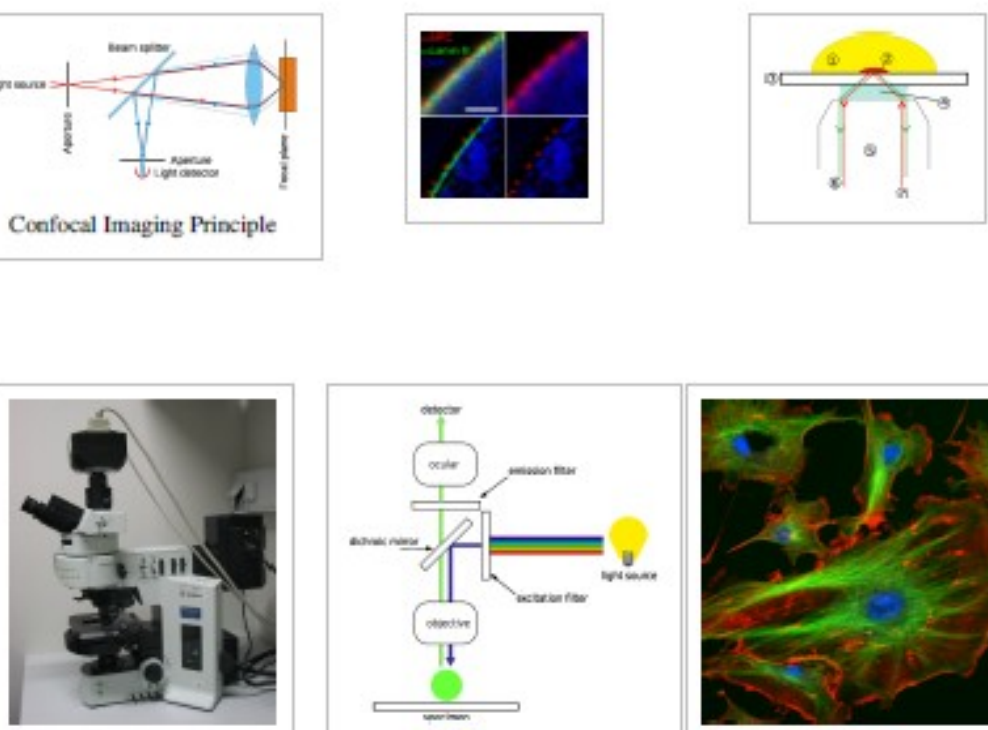

Fluorescence microscope
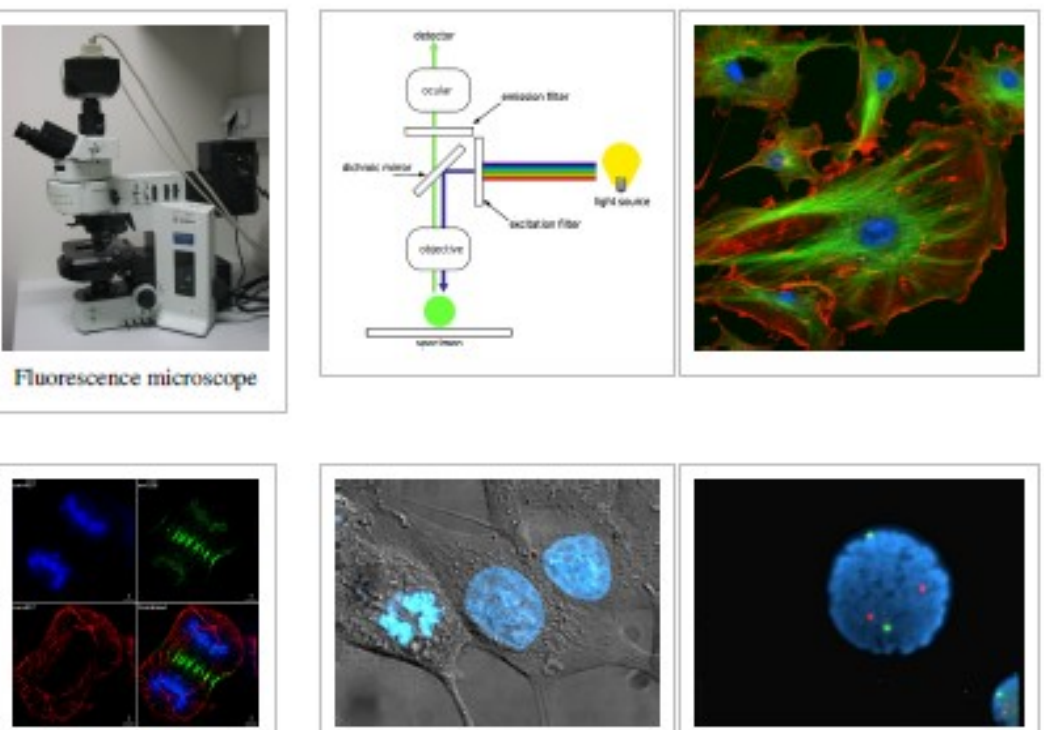

Dividing cell

fluorescence

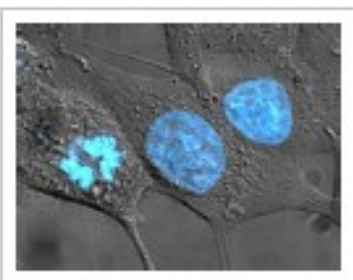

HeLa cancer cells

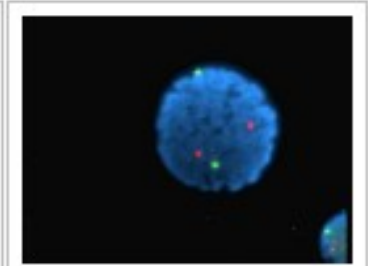

FISH fluorescence technique

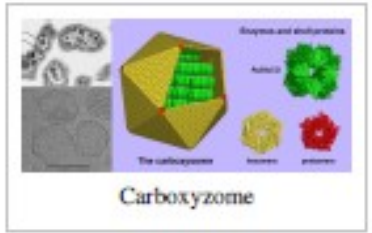




\section{JOURNALS SPECIALIZED IN FOOD PHYSICAL CHEMISTRY AND RELATED FIELDS}

- Journal of Agricultural and Food Chemistry

- AOCS Journal

- Biophysical Chemistry journal:

http://www.elsevier.com/wps/find/journaldescription.cws home/52499

- Magnetic Resonance in Chemistry

- Starke/ Starch Journal

- Journal of Dairy Science® (JDS): http://www.journalofdairyscience.org/

- Chemical Physics Letters

- International Journal of Research and Reviews in Applied Sciences

http://www.arpapress.com/

- Zeitschrift für Physikalische Chemie (1887)

- Biopolymers

- Journal of Food Science (IFT, USA)

- International Journal of Food Science \& Technology http://www.wiley.com/bw/journal.asp? $\underline{\text { ref }=0950-5423}$

- Macromolecular Chemistry and Physics (1947)

- Journal of the Science of Food and Agriculture (http://www.onlinelibrary.wiley.com/journal/10.1002/

(ISSN)1097-0010)

- Polymer Preprints http://www.polyacs.org/11.html (ACS)

- Integrative Biology- Journal of the Royal Society of Chemistry

http://pubs.rsc.org/en/journals/journalissues/ib

- Organic \& Biomolecular Chemistry http://pubs.rsc.org/en/journals/journalissues/ob

(An RSC Journal)

- Nature

- Journal of Biological Chemistry

- Proceedings of the National Academy of Sciences of the United States of America

\section{EXTERNAL LINKS}

- ACS Division of Agricultural and Food Chemistry (AGFD) (http:/ / agfd. sites. acs. org/ )

- American Chemical Society (ACS) (http:/ / portal. acs. org/ portal/ acs/ corg/ content)

- Institute of Food Science and Technology (IFST), (formerly IFT) (http:/ / www. ifst. org/ )

- Dairy Science and Food Technology (http:/ / www. dairyscience. info/ index. php)

- Physical Chemistry. (Keith J. Laidler, John H. Meiser and Bryan C. Sanctuary

http://www.mchmultimedia.com/store/Physical-Chemistry-ebooks.html

- The World of Physical Chemistry http://books.google.co.uk/books?id=01LRlPbH80cC

(Keith J. Laidler,1993)

- Physical Chemistry from Ostwald to Pauling http://books.google.co.uk/books?

$\underline{\mathrm{id}=1 \mathrm{UZjU} 2 W f L A o C}$

(John W. Servos, 1996)

- 100 Years of Physical Chemistry http://www.amazon.com/Years-Physical-Chemistry-RoyalSociety/dp/0854049878/ (Royal Society of Chemistry, 2004)

- The Cambridge History of Science: The modern physical and mathematical sciences http://books.google.co.uk/?id=B3WvWhJTTX8C (Mary Jo Nye, 2003).

\section{REFERENCES}

[1] John M. de Man.1999. Principles of Food Chemistry (Food Science Text Series), Springer Science, Third Edition

[2] John M. de Man. 2009. Food process engineering and technology, Academic Press, Elsevier: London and New

York, 1st edn.

[3] Pieter Walstra. 2003. Physical Chemistry Of Foods. Marcel Dekker, Inc.: New York, 873 pages.

[4] Physical Chemistry Of Food Processes: Fundamental Aspects.1992.van Nostrand-Reinhold vol.1., 1st Edition, 
[5] Henry G. Schwartzberg, Richard W. Hartel. 1992. Physical Chemistry of Foods. IFT Basic Symposium Series, Marcel Dekker, Inc.:New York, 793 pages

[6] I. C. Baianu, Editor. Physical Chemistry of Food Processes, Advanced Techniques, Structures and Applications.1994. van Nostrand-Reinhold vols.1-2., $1^{\text {st }}$ Edition, 998 pages; 3rd edn. Minuteman Press, 2010; vols. 2-3, fifth edition (in press)

[7] Pieter Walstra, Editor. 2003. Physical Chemistry Of Foods. Marcel Dekker, Inc.: New York, 873 pages

[8] Pieter Walstra, Editor. 2003. Physical Chemistry Of Foods. Marcel Dekker, Inc.: New York, 873 pages

[9] I. C. Baianu, Editor. Physical Chemistry Of Food Processes: Fundamental Aspects.1992.van Nostrand-Reinhold vol.1., 1st Edition,

[10] I. C. Baianu, Editor. Physical Chemistry of Food Processes, Advanced Techniques, Structures and Applications.1994. van Nostrand-Reinhold vols.1-2., $1^{\text {st }}$ Edition, 998 pages; 3rd edn. Minuteman Press, 2010; vols. 2-3, fifth edition (in press)

[11] First Nobel Prize for NMR in Physics, in 1952 http://www.nobelprize.org/nobel prizes/physics/laureates/952/

[12] ESR discovery in 1941, http://www.ismrm.org/12/aboutzavoisky.html

[13] Abragam, A.; Bleaney, B. Electron paramagnetic resonance of transition ions. Clarendon Press:Oxford, 1970, 1,116 pages.

[14] Physical Chemistry of Food Processes, Advanced Techniques, Structures and Applications.1994. van NostrandReinhold vols.1-2., ${ }^{\text {st }}$ Edition, 998 pages; 3rd edn. Minuteman Press, 2010; vols. 2-3, fifth edition (in press)

[15] Magde, D., Elson, E. L., Webb, W. W. Thermodynamic fluctuations in a reacting system: Measurement by fluorescence correlation spectroscopy,(1972) Phys Rev Lett, 29, 705-708.

[16] Ehrenberg, M., Rigler, R. Rotational brownian motion and fluorescence intensity fluctuations,(1974) Chem Phys, 4, 390-401.

[17] Elson, E. L., Magde, D. Fluorescence correlation spectroscopy I. Conceptual basis and theory, (1974) Biopolymers, 13, 1-27.

[18] Magde, D., Elson, E. L., Webb, W. W. Fluorescence correlation spectroscopy II. An experimental realization, (1974) Biopolymers, 13, 29-61.

[19] Thompson N L. 1991. Topics in Fluorescence Spectroscopy Techniques vol 1, ed J R Lakowicz (New York: Plenum); pp 337-78

[20] Gohlke, R. S. (1959). Time-of-Flight Mass Spectrometry and Gas-Liquid Partition Chromatography. Analytical Chemistry 31 (4): 535. doi:10.1021/ac50164a024.

[21] Gohlke, R; McLafferty, Fred W. (1993). Early gas chromatography/mass spectrometry. Journal of the American Society for Mass Spectrometry 4 (5): 367. doi:10.1016/1044-0305(93)85001-E.

[22] Alfsen, E.M.; Schultz, F.W. Geometry of State Spaces of Operator Algebras.; Birkhäuser: Boston - Basel Berlin, 2003.

[23] Anderson, P.W. Topology of Glasses and Mictomagnets. Lecture presented at the Cavendish Laboratory in Cambridge, UK, 1977.

[24] Baianu, I.C.; Editor. Quantum Algebra and Symmetry: Quantum Algebraic Topology, Quantum Field Theories and Higher Dimensional Algebra; PediaPress GmbH: Mainz, Second Edition, Vol. 1: Quantum Algebra, Group Representations and Symmetry; Vol. 2: Quantum Algebraic Topology: QFTs, SUSY, HDA; Vol. 3: Quantum Logics and Quantum Biographies, December 20, 2010; 1,068 pages total.

[25] Baianu, I.C. Structural Studies of Erythrocyte and Bacterial Cytoplasmic Membranes by X - ray Diffraction and Electron Microscopy. PhD Thesis; Queen Elizabeth College: University of London, UK, 1974.

[26] Baianu, I.C. $X$ - ray scattering by partially disordered membrane systems. Acta Cryst. A. 1978, 34, 751 - 753.

[27] Baianu, I.C.; Boden, N.; Levine, Y.K.;Lightowlers, D. Dipolar coupling between groups of three spin-1/2 undergoing hindered reorientation in solids. Solid State Comm. (1978), 27, 474 - 478.

[28] Baianu, I.C. Structural order and partial disorder in biological systems. Bull. Math. Biology. 1980, 42, 464 468.

[29] Baianu, I.C.; Boden N.; Levine Y.K.; Ross S.M. Quasi-quadrupolar NMR spin-echoes in solids containing dipolar coupled methyl groups. J. Phys. C: Solid State Phys. 1978, 11, L37--L41.

[30] Baianu, I.C.; Boden, N.; Lightowlers, D. NMR spin-echo responses of dipolar-coupled spin-1/2 triads in solids. J. Magnetic Resonance. 1981, 43, 101 - 111.

[31] Baianu, I.C.; Boden, N.; Mortimer, M.; Lightowlers, D. A new approach to the structure of concentrated aqueous electrolyte solutions by pulsed N.M.R. Chem. Phys. Lett. 1978, 54, 169 - 175.

[32] Baianu, I.C.; Glazebrook J.F.; Brown, R. A Non-Abelian, Categorical Ontology of Spacetimes and Quantum Gravity. Axiomathes 2007, 17, 353 - 408.

[33] Bellissard, J. K-theory of $\mathrm{C}^{*}$-algebras in solid state physics. Statistical Mechanics and Field Theory: Mathematical Aspects; Dorlas, T. C. et al., Editors; Springer Verlag. Lect. Notes in Physics, 1986, 257, 99 - 156. 
[34] Brown, R.; Glazebrook, J.F.; Baianu, I.C. A categorical and higher dimensional algebra framework for complex systems and spacetime structures. Axiomathes 2008, 17, 409 - 493.

[35] Georgescu, G. $N$-valued logics and Łukasiewicz--Moisil algebras. Axiomathes 2006, 16, 123-136.

[36] Hazewinkel, A. (Editor). Handbook of Algebra. Vol.4; Elsevier: St. Louis, Chatswood - Singapore, 2006.

[37] Hindeleh, A.M.; Hosemann, R. Paracrystals representing the physical state of matter. Solid State Phys. 1988, 21, 4155--4170.

[38]Hosemann, R.; and Bagchi, R.N. Direct analysis of diffraction by matter.; North-Holland Publs.: Amsterdam and New York, 1962.

[39] Hosemann, R.; Vogel W.; Weick, D.; Balta-Calleja, F.J. Novel aspects of the real paracrystal. Acta Cryst. A. 1981, 376, 85-91.

[40] Kauffman, L. Spin Networks and the Jones Polynomial. Twistor Newsletter, No.29 ; Mathematics Institute: Oxford, November 8th, 1989.

[41] Kauffman, L. SL(2) - Spin Networks. Twistor Newsletter, No.32 ; Mathematics Institute: Oxford, March 12th, 1989.

[42]Lawrence, R.L. Algebra and Triangle Relations. In: Topological and Geometric Methods in Field Theory; Editors: J. Michelsson and O.Pekonen; World Scientific Publishing,1992;pp.429-447. J.Pure Appl. Alg. 1995, 100, 245-251.

[43] Mackey, G.W. The scope and history of commutative and noncommutative harmonic analysis. History of Mathematics, Vol. 5; American Mathematical Society: Providence, RI; London Mathematical Society: London, 1992.

[44] Mott, N.F. Electrons in glass. Nobel Lecture, 11 pages, December 8, 1977.

[45] Mott, N.F. Electrons in solids with partial disorder. Lecture presented at the Cavendish Laboratory: Cambridge, UK, 1978.

[46] Mott, N.F.; Davis, E.A. Electronic processes in non-crystalline materials.; Oxford University Press: Oxford, 1971; 2nd ed., 1978.

[47] Mott, N.F.; et al. The Anderson transition. Proc. Royal Soc. London Ser. A. 1975, 345, 169 - 178.

[48] Paterson, A.L.T. The Fourier-Stieltjes and Fourier algebras for locally compact groupoids. In Trends in

Banach Spaces and Operator Theory, (Memphis, TN, 2001, Contemp. Math. 2003, 321, 223-237.

http://arxiv.org/abs/math.OA/0310138math.OA/0310138

[49] Plymen, R.J.; Robinson, P.L. Spinors in Hilbert space. Cambridge Tracts in Mathematics, Vol. 114; Cambridge University Press: Cambrige, 1994.

[50] Radin, C. Symmetries of Quasicrystals. Journal of Statistical Physics 1999, 95, Nos 5/6, 827--833.

[51] Ran, Y.; Wen, X.-G. Detecting topological order through a continuous quantum phase transition. Phys. Rev Lett., 2006, 96, 026802, 4 pages; http://arxiv.org/abs/cond-mat/0509155cond-mat/0509155

[52] Schwartz, L. Généralisation de la notion de fonction, de dérivation, de transformation de Fourier et applications mathématiques et physiques. Ann. Univ. Grenoble. Sect. Sci. Math. Phys. (N.S.) 1945, 21, 57-74.

[53] Schwartz, L. Théorie des distributions. Publications de l'Institut de Mathématique de l'Université de Strasbourg, no. IX--X; Hermann: Paris, 1966.

[54] Segal, I.E. Postulates for general quantum mechanics. Ann. of Math. (2) 1947, 4, 930--948.

[55] Weinberg, S. The quantum theory of fields., Vol. I. Foundations and Vol. II. Modern applications.; Cambridge University Press: Cambridge, 1996--1997.

[56] Weinberg, S. The quantum theory of fields. Vol. III. Supersymmetry; Cambridge University Press: Cambridge, 2005.

[57] Wen, X.-G. Quantum field theory of many--body systems -- from the origin of sound to an origin of light and electrons; Oxford University Press: Oxford, 2004.

[57] Westman, J.J. Harmonic analysis on groupoids. Pacific J. Math. 1968, 27, 621-632.

[58] Westman, J.J. Groupoid theory in algebra, topology and analysis; University of California at Irvine, 1971.

[59] Wickramasekara, S.; Bohm, A. Symmetry representations in the rigged Hilbert space formulation of quantum mechanics. J. Phys. A: Math. Gen. 2002, 35, 807-829. http://arxiv.org/abs/math-ph/0302018math-ph/0302018

[60] Abragam, A. The Principles of Nuclear Magnetism, Oxford University Press: Oxford, UK, 1961.

[61] Emch, G.G. Algebraic Methods in Statistical Mechanics and Quantum Field Theory. Dover Publications Inc: Mineola, New York, 2009 reprint.

[62] Connes, A. Noncommutative geometry; Academic Press, Inc.: San Diego, CA, 1994. 